\title{
The Non-Line-of-Sight Channel Modelling and Analysis for Airport Surface
}

\author{
Fengxun Gong ${ }^{1}$, Yemeng $\mathrm{Che}^{2}$ and Yanqiu $\mathrm{Ma}^{3}$ \\ College of Electronic and Information Engineering Civil Aviation University of China, Tianjin, 300300, China \\ 1'gfxcauc@eyou.com, ${ }^{2}$ cheyem@163.com, ${ }^{3}$ yqma@cauc.edu.cn
}

\begin{abstract}
A channel model has been established by analyzing the characteristics of the non-line-of-sight (NLOS) environment in a domestic airport surface and referencing mobile communication and air channel research. Through deducing the analytical formula of Doppler spectrum that incident angle in Gaussian distribution, we got the spread of frequency that produced by relative motion, and we assess the communication performance of the channel by contrasting the bit error rate of some channel. Channel evaluation also by analyzing channel capacity in different antenna configuration to compare priori knowledge. The simulation results show that the established channel model can accurately describe the characteristics of the non-line-of-sight environment in the airport surface, and it can be used for the design of multilateration (MLAT) system and the research of time difference of arrival (TDOA) localization algorithm.

Index Terms - Non-Line-of-Sight (NLOS), Channel Model, Multilateration (MLAT), Time Difference of Arrival (TDOA)
\end{abstract}

\section{Introduction}

For improving the positioning precision of MLAT system, it must solve the NLOS problem. The NLOS environment between the mobile station (MS) and base station (BS) can make excess delay of TDOA value, namely NLOS error. It can't eliminate NLOS error by improving the system of the measuring accuracy of TDOA receiver. The NLOS error is only affected by the wave channel.

The research on aviation channel especially NLOS channel suitable for airport surface at home and abroad is less than on wireless channel. In the aviation channel aspect, [1] analysis the characteristics of air channel. The authors do some improvement and implementation to the aviation channel statistical model based on the incident angle in [2]. In [3], a mixed application channel model about aviation and wireless communication is proposed, the model is used to simulate various fading signal. It analysis the unmanned air vehicle (UAV) in different flight status, proposed a statistical channel model that is applied to UAV in [4]. The interference between channels caused by multi-path and multiple antenna transceiver situations are introduced in [5] and [6]. The channels of above are all include LOS component, and has not test whether the channel applicable.

Referencing the channel model of above, and anglicizing the real NLOS airport surface environment, also combining with MLAT technology, a channel model is proposed and evaluated in this paper.

\section{Establishing Channel Model According to the Actual Environment}

The Description of Actual Airport Surface Environment. Multiple antenna communication combines antenna diversity and space-time code technology, which can effective against multipath fading and improve spectrum efficiency. The research about multiple antenna technology applied in aviation channel has started in recent years [7].

A domestic airport surface environment is shown in Fig.1. Scattering environment between the transmitting antenna array $T x$ and target receiving antenna array is made up of ground building vehicles and landing or take-off aircraft. The distance from MS to $\mathrm{BS}$ is $d$.

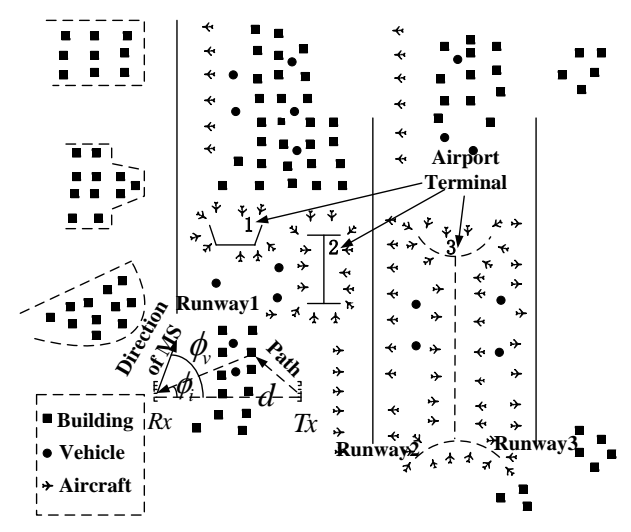

Fig. 1 The schematic diagram of an airport surface

Taking a single transmission path for example, we assume that the movement direction of MS is $\phi_{v}$. Signal will arrive at the target receiving antenna array in angle $\phi_{i}$ after reflection, diffraction or scattering of the scattering environment.

Channel Modeling. A channel model is proposed by referencing Fig. 1. Signal transmission between the master station and target uses antenna array. While the transmitting antenna element $j(j=1,2, \cdots, N t)$ arrive at the receiving antenna element $i(i=1,2, \cdots, N r)$, the channel impulse response vector $h_{i, j}(t, \tau)$ can be expressed as

$$
h_{i, j}(t, \tau)=\sum_{l=0}^{L} \beta_{i, j}^{l}(t) \cdot \alpha^{T}\left(\phi_{i}\right) \cdot \delta\left(\tau-\tau_{l}\right)
$$


In Eq. $1, \beta_{i, j}^{l}(t)$ denotes the fading coefficients of the path $l$, and it obeys the complex Gauss distribution. $L$ denotes the number of path, $\tau_{l}$ denotes the delay of path $l$, the value range of $\tau_{l}$ is $\left(0, \tau_{\max }\right)$, while $\tau_{\text {max }}$ is the RMS delay spread. The delay spread of this channel model is produced according to the Greenstein model [8], namely $\tau_{\text {max }}=T_{1} d^{\xi} y . T_{1}$ is the median delay spread while $d=1 \mathrm{~km}$. The value of $\xi$ is 0.5. $y$ denotes a random variable that obeys logarithmic normal distribution, and its standard deviation $\sigma_{y}=4 \mathrm{~dB}$. $\alpha\left(\phi_{i}\right)$ denotes the steering vector that sent by target antenna $i$ of which angle of arrival is $\phi_{i}$, and it can be written as

$\alpha\left(\phi_{i}\right)=\left[1, e^{\frac{2 \pi j}{\lambda} D \sin \phi_{i}}, e^{\frac{2 \pi j}{\lambda} 2 D \sin \phi_{i}}, \ldots, e^{\frac{2 \pi j}{\lambda}\left(N_{r}-1\right) D \sin \phi_{i}}\right]^{T}$

The spacing between the antenna is $D$, and $D=0.5 \lambda$, while $\lambda$ is the signal wavelength, and $\phi_{i} \in\left[-\frac{\pi}{2}, \frac{\pi}{2}\right]$.

The transfer function matrix of the channel can be expressed as

$$
\boldsymbol{H}=\left[h_{i, j}\right]_{N t \times N r}
$$

\section{The Derivation of Doppler Power Spectrum}

We suppose the speed of MS is $v$, and carrier frequency $f_{c}=c / \lambda$. Fig. 1 shows that the movement direction of MS is $\phi_{v}$. The Doppler frequency of path $l$ can be written as

$$
f_{i}=f_{d} \cos \left(\phi_{i}-\phi_{v}\right)+f_{c}
$$

The maximum Doppler frequency shift is $f_{d}$. Angle of arrive $\phi_{i}$ is

$$
\phi_{i}=\phi_{v} \pm \arccos \left|\frac{f_{i}-f_{c}}{f_{d}}\right|, \quad \phi_{i}^{>} \phi_{v}
$$

The number of incident wave is $N_{s}$, the probability density function (PDF) of the incident wave angle distribution is $p(\phi) . p(\phi) d \phi$ denotes the energy when the incident angle belongs to $[\phi, \phi+d \phi]$.

When the scattering distributed very intensive, $p(\phi) d \phi$ is close to continuous distribution. We use $\phi$ instead of $\phi_{i}, f$ instead of $f_{i}$. After derivation of Eq.4, we get

$$
d f=-f_{d} \sin \left(\phi-\phi_{v}\right) d \phi
$$

And from Eq.5, there is

$$
\sin \left(\phi-\phi_{v}\right)=\sqrt{1-\left(\frac{f-f_{c}}{f_{d}}\right)^{2}}
$$

We use $S(f)$ denotes the Doppler spectrum. Then

$$
S(f) d f=A[G(\phi) p(\phi)+G(-\phi) p(-\phi)] d \phi
$$

$A$ denotes the average receive power of the Omni-directional antenna. The antenna gain of direction $\phi$ is $G(\phi)$. We suppose antenna gain $\mathrm{G}(\phi)$ is constant on Omni-directional, namely $G(\phi)=1$. From Eq.5, Eq.6, Eq.7 and Eq.8 we get the complete expression of Doppler spectrum

$\mathrm{S}(f)=\frac{A[G(\phi) p(\phi)+G(-\phi) p(-\phi)]}{f_{d} \sqrt{1-\left(\frac{f-f_{c}}{f_{d}}\right)^{2}}}=\frac{p\left(\phi_{v}+\arccos \left(\frac{f-f_{c}}{f_{d}}\right)\right)+p\left(\phi_{v}-\arccos \left(\frac{f-f_{c}}{f_{d}}\right)\right)}{f_{d} \sqrt{1-\left(\frac{f-f_{c}}{f_{d}}\right)^{2}}},\left|f-f_{c}\right|<f_{d}$

For the convenience of the following derivation, we define

$$
\phi_{f}=\arccos \left(\frac{f-f_{c}}{f_{d}}\right), 0 \leq \phi_{f} \leq \pi
$$

The airport usually located in remote areas and suburban environment, and Gauss model is more practical than the uniform model. When the incident angle obeys Gauss distribution, its Standard deviation is $\sigma$, and the PDF is

$$
p(\phi)=K \exp \left(-\frac{\left(\phi-\phi_{0}\right)^{2}}{2 \sigma^{2}}\right) /(\sqrt{2 \pi} \sigma) ;-\pi+\phi_{0} \leq \phi \leq \pi+\phi_{0}
$$

$K$ denotes normalization coefficient and deduces that $K=1 / \operatorname{erf}(\pi / \sqrt{2} \sigma)$ while $\operatorname{erf}(\bullet)$ is error function. Bring Eq.11 to Eq.9, we get

$$
S(f)=\frac{K}{\sqrt{2 \pi} \sigma \cdot f_{d} \sqrt{1-\left(\frac{f-f_{c}}{f_{d}}\right)^{2}}} \cdot\left\{\exp \left[-\frac{\left(\phi_{v}+\phi_{f}-\phi_{0}\right)^{2}}{2 \sigma^{2}}\right]+\exp \left[-\frac{\left(\phi_{v}-\phi_{f}-\phi_{0}\right)^{2}}{2 \sigma^{2}}\right]\right\} ;\left|f-f_{c}\right|<f_{d}
$$

\section{Simulation Results with Analysis}

In order to evaluate the established channel model in this paper, we did four kinds of simulation as below. The general parameters of the simulation include the carrier frequency 2 $\mathrm{GHz}$ and the speed of MS $200 \mathrm{~km} / \mathrm{h}$.

Simulation of Doppler Power Spectrum. Suppose the incident wave obeys the Gaussian distribution, average incident angle $\phi_{0}=0^{\circ}$ and the movement direction of the target is $0^{\circ}, 90^{\circ}$ and $180^{\circ}$. We get the Doppler spectrum in different direction as follows. 


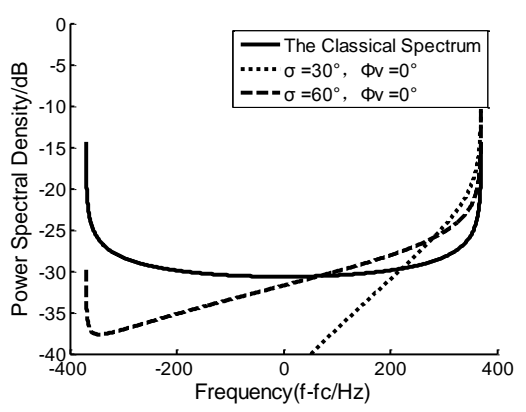

Fig. $2 \phi_{v}=0^{\circ}$

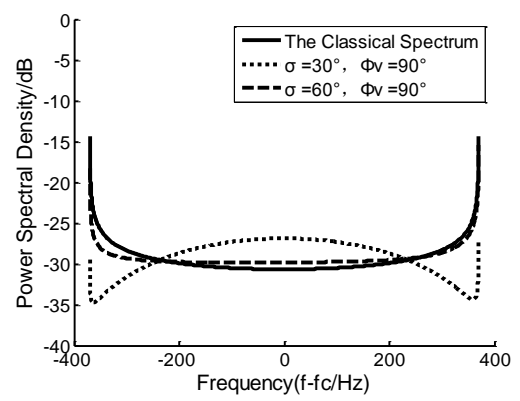

Fig. $3 \phi_{v}=90^{\circ}$

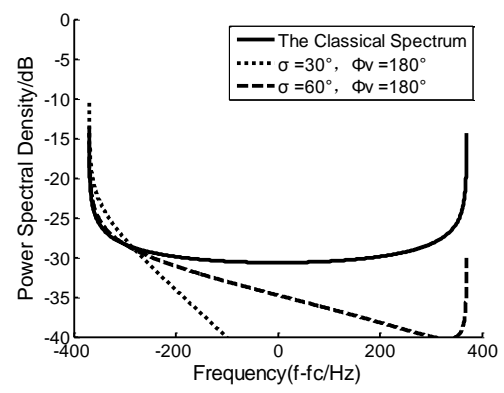

Fig. $4 \phi_{v}=180^{\circ}$

Fig. 2 shows that when the movement direction is $0^{\circ}$, MS move towards BS. When $\sigma$ is small, it means the incident expansion angle is small. At this time the scatterer are mainly distributed between the MS and BS, and the power spectrum energy is mainly distributed in the positive frequency shift. AS $\sigma$ becomes bigger, the incident expansion angle becomes bigger. So the scatterer behind BS increases as the energy in the negative frequency shift gradually increases.

Fig. 3 shows that when the movement direction is $90^{\circ}$, MS moves perpendicular to the connection between MS and BS. When $\sigma$ is small, the scattering object are relatively concentrated near the average incidence angle. Small frequencies shift have larger energy while large frequencies shift with less energy. AS $\sigma$ becomes bigger, scattering distribution tends to be uniform distribution of $[-\pi, \pi]$ and power spectrum approaches to the classic spectrum.

Fig. 4 tells that when the movement direction is $180^{\circ}$, MS deviate from BS. The Doppler power spectrum is mainly concentrated in the negative half shaft.

Simulation of Bit Error Rate (BER). Rayleigh channel is a commonly used NLOS channel. We calculate the BER of our channel and compare it to the Rayleigh channel and the channel in [5]. The result is shown in Fig. 5.

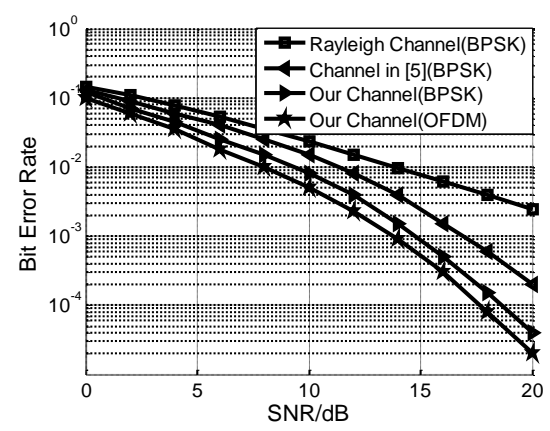

Fig. 5 The comparison of different channels.

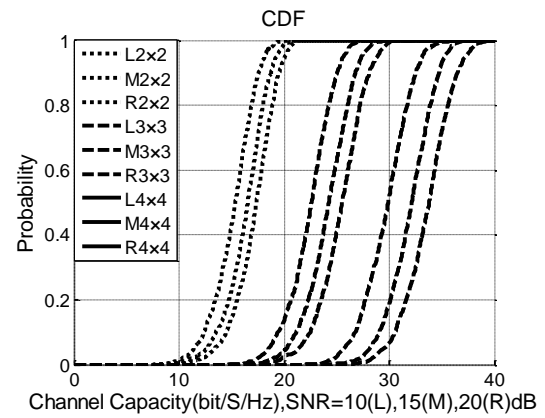

(a) Total channel capacity.

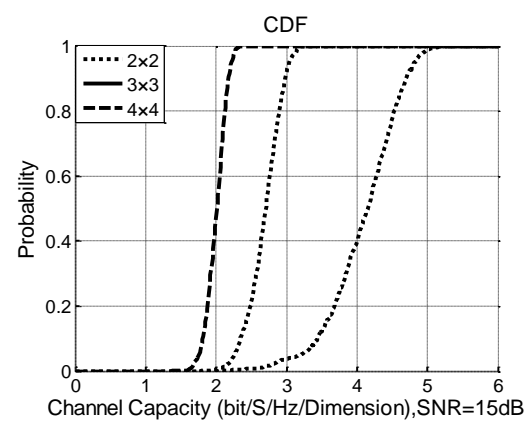

(b) Channel capacity per dimension.

Fig. 6 The CDF of channel capacity.

Fig. 5 shows that the channel BER decreases along with the signal-to-noise ratio (SNR) increases. The BER of our channel is small than the Rayleigh channel and the channel in [5], so our channel has good communication performance and suitable for the analysis of NLOS environment. And we also see that the OFDM modulation has lower BER than the BPSK modulation.

Simulation of Cumulative Distribution Function (CDF) of Channel Capacity. The comparison of the total channel capacity and the channel capacity per dimension in 
different antenna configuration is shown in Fig. 6. The different antenna configuration is $2 \times 2,3 \times 3$ and $4 \times 4$.

From Fig. 6, the channel capacity increases as the SNR increases. As the number of antenna increases, the total channel capacity increases while the channel capacity per dimension decreases. Because the more the number of antenna is, the larger the mutual correlation of each antenna becomes. It makes the rank of the channel transfer function matrix with additional loss, and finally leads to the drop of the channel capacity per dimension. This result is the same as Fig. 12 in [9]. So our channel coincides with the priori knowledge.

Simulation of Geometric Dilution of Precision (GDOP) in Different Channels. We suppose the height of the target is $2 \mathrm{~km}$, and use the Star-model Station. Then we get the coverage of positioning precision in different channels as below.

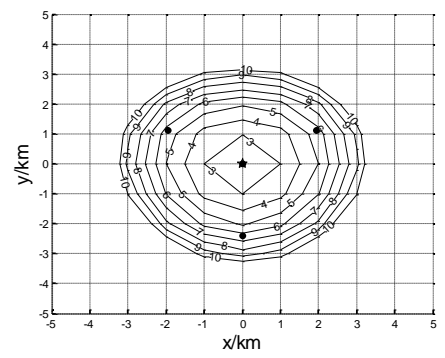

(a) Rayleigh channel.

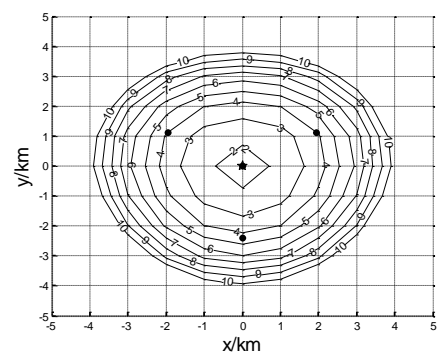

(b) The channel in [5].

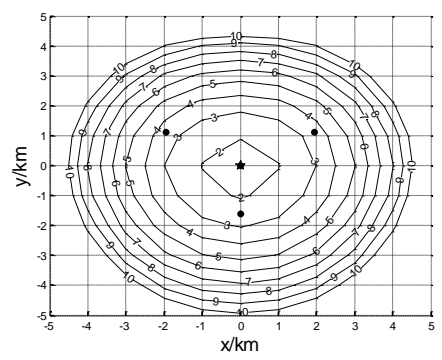

(c) Our channel.

Fig. 7 The GDOP contour line in different channels.
Different channels produce different TDOA error that decline the precision of the location algorithm. We get the GDOP contour line with the same embattling mode. From Fig. 7 , the positioning coverage of our channel is larger than the Rayleigh channel and the channel in [5]. So our channel is suitable for the analysis of location algorithm.

\section{Conclusions}

Establishment of NLOS propagation channel model that conforms to the real environment has important significance for improving the precision of the positioning system. According to the NLOS problem in MLAT system, a channel model is proposed after the analysis of the real airport surface environment. The established channel is based on the distribution of the scattering object, and the construction method is clear. Simulations and the analysis show that the model is suitable for NLOS environment and it can well describe the condition of Doppler frequency shift caused by relative motion. It has good communication performance and the increase or decrease trend of channel capacity in different antenna configuration coincide with the prior knowledge. And it can be used for the design of MLAT system and the research of TDOA localization algorithm.

\section{Acknowledgments}

This work is financially supported by the National Natural Science Foundation of China (No. 61079008, U1233112), the Application Basis and Front Technology of Tianjin (No. 11JC2DJC25200) and Fundamental Research Funds for The Central Universities (No. ZXH2011B001; ZXH2011D011).

\section{References}

[1] P.A. Bello: IEEE Trans on Communication, Vol. 21 (1973) No.5, P.548.

[2] Q.M. Zhu, D.Z. Xu and W.H. Lu: Journal of Applied Sciences, Vol.27 (2009) No.6, P. 569.

[3] Q.H. Pan, L.M. Zhao and M. Xu: Computer Simulation, Vol. 28 (2011) No.6, P.52.

[4] Z. Tan and X.L. Zhang: Journal of Beijing University of Aeronautics and Astronautics, Vol. 36 (2010) No.10, P. 1171.

[5] M.A. Nguyen and A. I. Zaghloul: IEEE Trans on Vehicular Technology, Vol. 56 (2007) No.2, P. 837.

[6] M.A. Jensen, M.D. Rice and A.L. Anderson: IEEE Trans on Aerospace and Electronic Systems, Vol. 43 (2007) No.1, P. 262.

[7] N. Czink, A. Richter, E. Bonek and J.P. Nuutinen, J. Ylitalo: IEEE Trans on Vehicular Technology, Vol. 66 (2007) No.6, P. 874.

[8] L. J. Greenstein et.al. IEEE Trans on Vehicular Technology, Vol. 46 (1997) No.2, P. 477.

[9] K. Yu, M. Bengtsson, B. Ottersten, D. McNamara, P. Karlsson and M. Beach: IEEE Trans on Vehicular Technology, Vol. 53 (2004) No.3, P.655. 